\title{
Quaderni
}

QUADERNI Communication, technologies, pouvoir

81 | Printemps 2013

L'humain médicament

\section{Entretien avec Sophie Samuel-Lucas, Pôle Thérapies innovantes et produits issus du corps humain à l'ANSM}

Aurélie Mahalatchimy et Emmanuelle Rial-Sebbag

\section{(2) OpenEdition \\ Journals}

Édition électronique

URL : http://journals.openedition.org/quaderni/718

DOI : $10.4000 /$ quaderni.718

ISSN : 2105-2956

Éditeur

Les éditions de la Maison des sciences de l'Homme

Édition imprimée

Date de publication : 5 juin 2013

Pagination : 89-94

Référence électronique

Aurélie Mahalatchimy et Emmanuelle Rial-Sebbag, « Entretien avec Sophie Samuel-Lucas, Pôle

Thérapies innovantes et produits issus du corps humain à I'ANSM », Quaderni [En ligne],

81 | Printemps 2013, mis en ligne le 05 mai 2015, consulté le 21 avril 2019. URL : http://

journals.openedition.org/quaderni/718; DOI : 10.4000/quaderni.718 


\section{$D$ ossier}

\section{Entretien avec} Sophie

Samuel-Lucas,

Pôle Thérapies innovantes et produits issus

du corps humain

à l'ANSM

Aurélie

Mahalatchimy

Doctorante en droit, UMR U 1027, Inserm, Université Paul Sabatier - Toulouse III ; IRDEIC, Université

Toulouse 1 - Capitole; Chercheure associée au laboratoire PACTE Département Politique,

Organisations UMR 5194

Emmanuelle Rial-Sebbag

Chargée de Recherches UMR U 1027, Inserm, Université Paul Sabatier - Toulouse III
L'Agence Nationale de Sécurité du Médicament et des produits de santé (ANSM) est en charge de l'évaluation et de la surveillance des produits de santé, y compris des produits de thérapies innovantes. Elle exerce également les missions d'inspection et de contrôle des établissements qui développent ces produits. Enfin, elle a mis en place une « cellule d'accompagnement de l'innovation ».

Quel est l'apport du règlement européen sur les médicaments de thérapie innovante?

À titre principal, s'il a clarifié la réglementation de ces produits pour les industriels, il est arrivé un peu tôt au regard du développement de ces produits. C'est pourquoi deux Autorisations de Mise sur le Marché (AMM) européennes seulement ont été délivrées en application de ce règlement (une pour un médicament de thérapie cellulaire en 2009, Chondrocelect, et l'autre pour un médicament de thérapie génique en 2012, Glybera). Encore peu d'établissements pharmaceutiques sont actifs dans ce domaine car les preuves d'efficacité concrètes manquent.

En revanche, pour l'ANSM, qui évalue depuis longtemps ce type de produit et possède donc déjà l'expertise dans ce domaine, il implique uniquement de s'adapter au nouveau cadre réglementaire.

Quels sont les changements au niveau institutionnel ?

Au niveau de l'Union européenne, le Comité des thérapies innovantes (CAT), au sein duquel un représentant français a été nommé, a été mis en 
place. Il est composé d'experts dans le domaine. Donc l'évaluation est plus adaptée aux particularités des médicaments de thérapie innovante.

Au niveau national, il existait jusqu'à fin 2012 : deux groupes de travail compétents en recherche biomédicale (l'un pour la thérapie génique, l'autre pour la thérapie cellulaire), ces groupes intervenant pour l'évaluation de ces produits et la Commission de thérapie génique et cellulaire qui évaluait notamment les cellules souches hématopoïtiques utilisées dans la restauration de l'hématopoïèse. Ces instances viennent d'être remplacées, dans le cadre de la réorganisation des instances consultatives de l'Agence, par des commissions généralistes, couvrant l'ensemble des produits de santé et par un groupe d'experts sur les thérapies innovantes qui sera consulté ponctuellement pour des situations particulières, principalement pour certains cas de thérapie génique, de thérapie cellulaire ou de greffe de la face.

La « cellule innovation " sera conservée. Elle continuera d'accompagner les porteurs de projet en amont d'une procédure d'essais cliniques, d'avis scientifiques ou en phase pré-AMM pour leurs questions réglementaires (ex. classification d'un produit de santé comme dispositif médical, médicament de thérapie innovante ou préparation de thérapie cellulaire). Dans le cadre de certaines de ces réunions " innovation 》, des experts externes peuvent également être consultés sur des cas particuliers. Dans le cadre des rencontres «innovation», pour les projets les plus complexes, les porteurs de projet peuvent être amenés à rencontrer plusieurs fois l'ANSM au fur et à mesure de l'avancement de leur projet, y compris dans le domaine des thérapies géniques et cellulaires. Quelles sont les difficultés rencontrées pour l'évaluation de ces produits?

Pour les préparations qui utilisent des cellules souches hématopoiétiques, les procédés ne sont pas très compliqués. L'évaluation est donc très standardisée. Pourtant, il ne s'agit pas d'un procédé générique. Mais s'il y a des différences, celles-ci ne sont pas majeures. Cependant, pour les autres produits, l'évaluation est plus complexe car de nouveaux procédés différents apparaissent régulièrement. Finalement, si tout n'est pas standardisé, la méthodologie d'évaluation l'est.

\section{Le règlement européen modifie-t-il les caté- gories de produits de thérapie innovante?}

Oui, il clarifie ces catégories, en particulier pour les produits dits frontières (borderlines).

Au niveau de l'Agence Européenne des médicaments (EMA), la classification des produits suscite de nombreuses discussions. Mais lorsque le CAT adopte une recommandation sur la classification de ces produits, elle devrait être respectée car les États membres sont représentés au sein de ce comité. Même si l'ANSM n'est pas forcément d'accord avec certaines recommandations qui sont adoptées à la majorité, elle a fait le choix de les respecter. Le domaine est suffisamment complexe pour qu'elle n'ait pas envie de faire des particularités nationales.

Au niveau français, certaines préparations de thérapie cellulaire répondent désormais à la définition des médicaments de thérapie innovante. L'établissement doit décider s'il veut 
développer son produit à l'échelle artisanale (il devra alors respecter le régime juridique national applicable aux médicaments de thérapie innovante préparés ponctuellement (MTI-PP) dont la mise en place est en cours au niveau national) ou à une échelle plus industrielle (il devra alors respecter le régime juridique européen applicable aux médicaments de thérapie innovante (MTI)). Nous sommes donc dans une période transitoire au cours de laquelle la réglementation nationale se met en place. Elle doit permettre aux établissements développant ce type de produits de faire des choix et de trouver des solutions en termes structurels pour se conformer à la réglementation.

\section{Cette réorganisation remet-elle en cause le principe de non-commercialisation des éléments du corps humain ?}

Avec le nouveau règlement européen, certaines préparations de thérapie cellulaire qui n'étaient pas qualifiées de médicaments en droit français sont amenées à changer de catégorie juridique pour devenir des médicaments de thérapie innovante.

Les établissements, publics ou privés, peuvent développer ce type de produits, et transformer ces éléments du corps humain. Mais actuellement ce domaine est dominé par le secteur public. En effet, initialement cette recherche s'y est développée alors que l'industrie pharmaceutique et les laboratoires privés y sont peu ou pas représentés. Aujourd'hui, la recherche française publique est très riche et se valorise à travers l'émergence de start-up. Mais cela ne veut pas dire que l'éthique relève du secteur public et non du secteur privé.
De plus, en raison de cette transformation, le produit de départ n'est pas le même qu'à l'arrivée, c'est à l'issue de cette transformation que l'on obtient le médicament. On a eu de réelles difficultés sur ce point car, en 1996, la loi précisait qu'il y avait d'un côté les médicaments, et de l'autre les cellules humaines. Ceci a entraîné des difficultés d'interprétation car on ne savait pas comment faire la différence entre les deux. Dans tous les cas, si le patient a besoin que ses cellules soient transformées pour être soigné, cela reste un bénéfice pour le patient. D'autant plus que la plupart des produits sont d'origine autologue. Ce sont donc les cellules d'un patient qui sont transformées et qui retournent chez ce même patient sous une "forme " plus adaptée à sa prise en charge thérapeutique. Lorsque le produit est d'origine allogénique, le donneur de cellules est informé sur l'utilisation future du produit qui en sera dérivé et on lui demande son consentement.

Ces questions ont largement freiné le développement de ces produits. Si le conflit de bioéthique a toujours été là, aujourd 'hui, il s'est un peu déplacé. Il n'est plus vraiment lié à la question des cellules versus les médicaments, mais à celle de l'utilisation des cellules souches embryonnaires humaines (CSEh) car elles proviennent d'un embryon. Ces discussions sont très présentes aux États-Unis où les premiers essais cliniques ont été mis en place; mais assez peu en France car nous n'avons pas encore beaucoup de produits.

\section{L'utilisation des CSEh soulève-t-elle des difficultés particulières pour l'ANSM ?}

L'ANSM est approchée par des équipes fran- 
çaises qui vont bientôt déposer des demandes impliquant des CSEh. Cette question a suscité une grande discussion au moment de la révision de la loi sur la bioéthique. L'Agence de la biomédecine peut autoriser des protocoles de recherches sur les CSEh par dérogation à condition que le demandeur démontre notamment que toutes les autres alternatives ont été épuisées. Une fois les premiers stades pré-cliniques passés, la loi de bioéthique allait-elle bloquer les recherches biomédicales au niveau de l'ANSM ? Ce point a été clarifié, et effectivement, il est possible de faire des préparations de thérapie cellulaire à partir de CSEh.

Pour les médicaments réglementés au niveau de l'Union européenne, il n'y a pas de différence en fonction de l'origine des cellules. Mais le règlement européen concernant les MTI comporte une clause qui permet aux États Membres de s'opposer à la commercialisation de médicaments issus de CSEh sur leur territoire. Pourtant, cela pose des problèmes au niveau éthique car ces produits seront souvent proposés lorsqu'il n'y aura pas d'alternative thérapeutique conventionnelle.

Cela étant, ces produits ne soulèvent pas de problème particulier en termes d'évaluation. Mais pour l'instant, l'ANSM est seulement approchée dans le cadre de l'accompagnement à l'innovation et non dans le cadre de protocole d'essais cliniques. Les demandes sont peu nombreuses, deux ou trois demandes concernant les CSEh et les cellules pluripotentes induites (IPS).

\section{Quelles sont les actions menées en termes de communication vers les parties intéressées ?}

Par rapport aux médicaments conventionnels, la réglementation des produits de thérapie innovante est assez compliquée pour les demandeurs et cela peut être un frein au développement. C'est pourquoi, l'ANSM a mis en place des réunions dans le cadre de la cellule innovation. Elle a mis en ligne, sur le site de l'Agence, des présentations, organisé des réunions, participé à des congrès, donné des cours pour expliquer la réglementation de ces produits.

Malgré ces actions de l'ANSM, certains demandeurs contactent la cellule innovation assez tardivement alors que ces contacts pourraient leur permettre d'identifier des difficultés qu'ils pourraient rencontrer lors du développement de leur produit. De même, l'Agence a intérêt à aller dans le sens d'un accompagnement pour les produits innovants car cela lui permet à elle aussi d'anticiper les difficultés scientifiques ou réglementaires de ces projets innovants et ainsi de mieux y répondre.

Pour les mêmes raisons, l'ANSM peut conseiller aux développeurs de produits innovants qui tombent dans le champ des médicaments réglementés au niveau européen, d'aller discuter rapidement avec l'EMA, soit par le biais des avis scientifiques, soit par le biais de réunions de concertation. L'idée est d'avancer ensemble pour le bénéfice des patients qui utiliseront ces produits.

Par ailleurs, il est difficile pour l'ANSM de fournir des données chiffrées par type de produits notamment dans le domaine extrêmement concurrentiel des CSEh.

De nouvelles adaptations sont-elles prévues? 
La Commission européenne doit bientôt publier un rapport sur l'application du règlement. Des choses vont certainement être revues ou améliorées.

Au niveau français, pour les MTI-PP, l'ANSM essaie de mettre en place un référentiel assez proche de celui des MTI réglementés au niveau européen. En effet, des produits développés à petite échelle dans un premier temps pourraient, selon la pathologie ou l'évolution du procédé de production notamment, évoluer vers des MTI développés à grande échelle. L'idée est donc de faciliter un tel cheminement.

Les MTI-PP ne sont pas encadrés au niveau européen. Donc leur réglementation n'est vraiment pas harmonisée entre les différents États membres. Pourtant, lorsque les représentants nationaux se retrouvent à l'EMA, ils en profitent pour discuter de la mise en place de leur règlementation nationale pour ces produits. Sur ce point, les agences nationales sont très sollicitées par la Commission européenne pour savoir comment les États ont réglementé ces produits, et si des autorisations ont été délivrées. Les États sont donc très encadrés par la Commission européenne qui ne veut pas que ces règlementations nationales fassent dévier le règlement européen sur les MTI de son objectif, c'est-à-dire que des produits accèdent seulement à un marché national alors qu'ils devraient relever d'une AMM européenne centralisée et accéder ainsi au marché de l'ensemble des États Membres de l'UE pour le bénéfice d'un plus grand nombre de patients.

Par ailleurs, la catégorie des préparations de thérapie génique et de thérapie cellulaire xéno- génique, qualifiées de médicaments en droit français mais préparées à petite échelle, devrait être amenée à disparaître (mais cela nécessite une révision de la loi). Elles devraient tomber dans le champ des MTI-PP, ce qui clarifierait le cadre réglementaire. Les spécificités sont minimes, elles ne nécessitent pas le maintien d'une catégorie et d'un régime juridiques particuliers. Pour la thérapie génique et la thérapie cellulaire xénogénique, on aura donc deux cadres juridiques possibles : soit les MTI développés à l'échelle industrielle et réglementés au niveau européen, soit les MTI-PP réglementés au niveau national.

Par contre, pour la thérapie cellulaire humaine, la catégorie des préparations de thérapie cellulaire réglementées au niveau national et non qualifiées de médicaments, est maintenue. Ces préparations doivent respecter la législation européenne (directive européenne 2004/23/CE) fixant des normes de qualité et de sécurité pour les cellules et tissus à finalité thérapeutique.

Cette catégorie des préparations de thérapie cellulaire humaine comprendra d'autres types de cellules que les cellules souches hématopoïétiques telles que les îlots de Langerhans.

Les cellules humaines peuvent être qualifiées de médicaments, ou de préparations. Lorsqu'elles sont préparées au bloc opératoire par un dispositif médical selon un procédé simple, elles ne tombent pas dans le champ de ces deux réglementations. Alors que lorsqu'il s'agit de gènes, on est toujours dans le domaine du médicament, cela ne soulève pas trop de questions. Le problème de délimitation se situe plus entre médicament de thérapie génique versus médicament classique 
ou vaccin par exemple.

\section{Quelle est l'avenir des produits de thérapie innovante?}

Aujourd'hui, c'est assez difficile à dire car tant qu'un produit n'a pas démontré son efficacité, les industriels ne vont pas investir. Et, on ne sait pas vraiment expliquer les échecs. Pour la thérapie cellulaire, il s'agit peut-être de produits mal caractérisés dont les plans de développement sont mal définis. Mais il y a un énorme potentiel. En France, on a une très bonne recherche publique avec des chercheurs très innovants qui ont le savoir-faire et sont à l'origine de la majorité des projets. Les industriels regardent cela de près. Mais nous sommes peut-être un peu différents par rapports à d'autres États. Par exemple en Allemagne, il y a beaucoup plus de structures industrialisées qu'en France où le développement reste majoritairement public.

Nous sommes dans une période charnière où le mouvement est à double sens. Les institutionnels et les chercheurs vont vers une professionnalisation du développement des produits, c'est-àdire vers le secteur privé qui dispose des moyens financiers et matériels nécessaires à l'élaboration d'un plan de développement concret pour ces produits. Et les industriels de la pharmacie observent pour savoir quel produit ils peuvent racheter, et cela plus sérieusement grâce au règlement européen qui a apporté des clarifications réglementaires.

[ Cette publication a été approuvée par l'ANSM. ] 